10. Atak ZK, Gianfelici V, Hulselmans G, De Keersmaecker K, Devasia AG, Geerdens E, et al. Comprehensive analysis of transcriptome variation uncovers known and novel driver events in Tcell acute lymphoblastic leukemia. PloS Genet. 2013;9:e1003997.

11. Treanor LM, Zhou S, Janke L, Churchman ML, Ma Z, Lu T, et al. Interleukin-7 receptor mutants initiate early $\mathrm{T}$ cell precursor leukemia in murine thymocyte progenitors with multipotent potential. J Exp Med. 2014;211:701-13.

12. Lin YW, Slape C, Zhang Z, Aplan PD. NUP98-HOXD13 transgenic mice develop a highly penetrant, severe myelodysplastic syndrome that progresses to acute leukemia. Blood. 2005;106:287-95.

13. Hu Y, Smyth GK. ELDA: extreme limiting dilution analysis for comparing depleted and enriched populations in stem cell and other assays. J Immunol Methods. 2009;347:70-8.

14. Bushman F. AllOnco. http://www.bushmanlab.org/links/genelists (2016). Accessed 19 December 2016.

15. Zhao M, Kim P, Mitra R, Zhao J, Zhao Z. TSGene 2.0: an updated literature-based knowledgebase for tumor suppressor genes. Nucleic Acids Res. 2016;44:D1023-31.

\title{
Lymphoma
}

\section{Ibrutinib versus temsirolimus: 3-year follow-up of patients with previously treated mantle cell lymphoma from the phase 3, international, randomized, open-label RAY study}

\author{
S Rule $\mathbb{D}^{1} \cdot \mathrm{W}$ Jurczak $^{2} \cdot \mathrm{M}$ Jerkeman $^{3} \cdot \mathrm{C}$ Rusconi $^{4} \cdot \mathrm{M}$ Trneny $^{5} \cdot \mathrm{F}$ Offner $^{6} \cdot \mathrm{D}$ Caballero $^{7} \cdot \mathrm{C}$ Joao $^{8}$. \\ M Witzens-Harig ${ }^{9} \cdot$ G Hess $^{10} \cdot$ I Bence-Bruckler $^{11} \cdot \mathrm{S}-\mathrm{G}$ Cho $\mathbb{D}^{12} \cdot \mathrm{C}$ Thieblemont $^{13} \cdot$ W Zhou $^{14} \cdot$ T Henninger $^{14}$. \\ $\mathrm{J} \mathrm{Goldberg}^{14} \cdot \mathrm{J} \mathrm{Vermeulen}^{15} \cdot$ M Dreyling $^{16}$
}

Received: 8 September 2017 / Revised: 21 November 2017 / Accepted: 22 December 2017 / Published online: 2 February 2018

(c) The Author(s) 2018. This article is published with open access

Mantle cell lymphoma (MCL) is an aggressive B-cell malignancy with a reported median overall survival (OS) of 3-5 years [1]. Most patients relapse after first-line therapy and have a poor prognosis [1]. Regulatory approval of ibrutinib has provided a much needed therapeutic option for patients with relapsed or refractory (R/R) MCL [2], with ibrutinib becoming a preferred standard of care in current guidelines [3, 4]. The randomized, open-label phase 3 RAY study (NCT01646021) was key in confirming the efficacy and safety of ibrutinib, with ibrutinib $(N=139)$ showing

S Rule

Simon.Rule@nhs.net

1 Plymouth University Medical School, Plymouth, UK

2 Department of Hematology, Jagiellonian University, Krakow, Poland

3 Skånes University Hospital, Lund University, Lund, Sweden

4 Hematology Division, Hematology and Oncology Department, Niguarda Cancer Center, Niguarda Hospital, Milan, Italy

5 Ist Dept Medicine, Charles University General Hospital, Prague, Czech Republic

6 Departement Oncologie, UZ Gent, Ghent, Belgium

7 Instituto Biosanitario de Salamanca, Hospital Clinico Universitario Salamanca, Salamanca, Spain

8 Present address: Institutto Português de Oncologia de Lisboa, significantly improved progression-free survival (PFS) versus temsirolimus $(N=141)$ (primary analysis [20-month followup]: 14.6 vs. 6.2 months, hazard ratio [HR] $0.43,95 \%$ confidence interval [CI]: 0.32-0.58) [5]. Here, we report extended follow-up data from the final analysis of the RAY study.

At this final analysis, after an almost doubled median study follow-up of 38.7 months, 33 patients (24\%) in the ibrutinib group and no patients in the temsirolimus group remained on initially randomized treatment. Crossover to ibrutinib from the temsirolimus group was permitted for

Portugal and Champalimaud Centre for the Unknown,

Hematology, Lisbon, Portugal

9 Klinikum der Ruprechts-Karls-Universität Heidelberg, Med. Klinik u. Poliklinik V, Heidelberg, Germany

10 Department of Hematology, Oncology and Pneumology, University Medical School of the Johannes Gutenberg University, Mainz, Germany

11 The Ottawa Hospital, General Campus, Ottawa, ON, Canada

12 Seoul St. Mary's Hospital, Seocho-gu, Seoul, South Korea

13 APHP, Saint-Louis Hospital, Hemato-oncology, Diderot University, Paris, France

14 Janssen Research \& Development, Raritan, NJ, USA

15 Janssen Research \& Development, Leiden, The Netherlands

16 Department of Medicine III, Klinikum der Universität München, LMU, Munich, Germany 
A

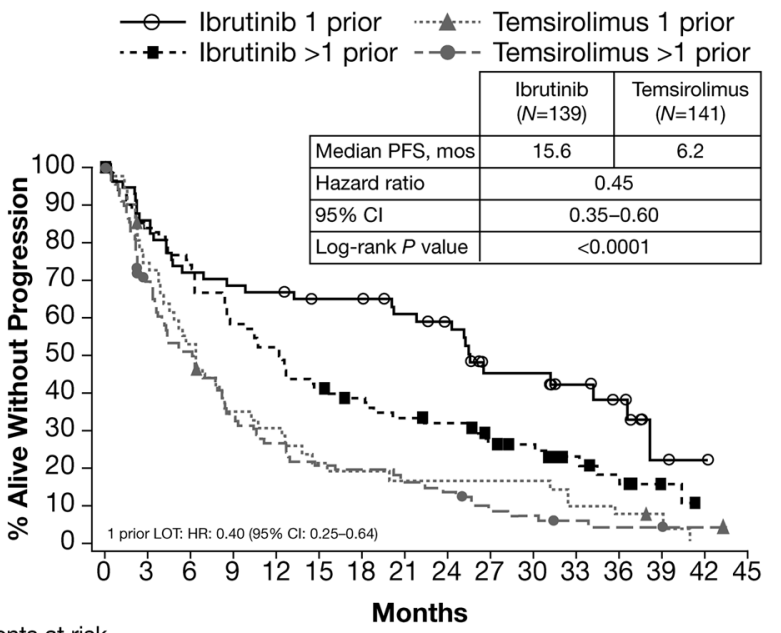

Patients at risk

Ibrutinib 1 prior

$\begin{array}{llllllllllllllll}57 & 49 & 41 & 39 & 38 & 34 & 33 & 30 & 27 & 15 & 15 & 11 & 8 & 2 & 1 & 0\end{array}$

Temsirolimus 1 prior

$\begin{array}{llllllllllllllll}50 & 34 & 24 & 15 & 13 & 9 & 8 & 7 & 7 & 7 & 7 & 4 & 3 & 1 & 1 & 0\end{array}$

lbrutinib $>1$ prior

$\begin{array}{llllllllllllllll}82 & 68 & 59 & 47 & 42 & 33 & 29 & 25 & 23 & 17 & 15 & 10 & 6 & 3 & 0 & 0\end{array}$

Temsirolimus $>1$ prior

$\begin{array}{llllllllllllllll}91 & 59 & 43 & 27 & 22 & 17 & 16 & 13 & 11 & 6 & 5 & 3 & 2 & 1 & 0 & 0\end{array}$

C

- Ibrutinib 1 prior $\quad . .-$ Ibrutinib $>1$ prior

\begin{tabular}{|l|c|c|c|}
\hline Median DOR, mos & ITT & 1 prior & $>1$ prior \\
\hline All responders & 23.1 & 32.1 & 13.5 \\
\hline CR & 35.6 & 35.6 & 32.2 \\
\hline PR & 12.1 & 22.3 & 10.0 \\
\hline
\end{tabular}

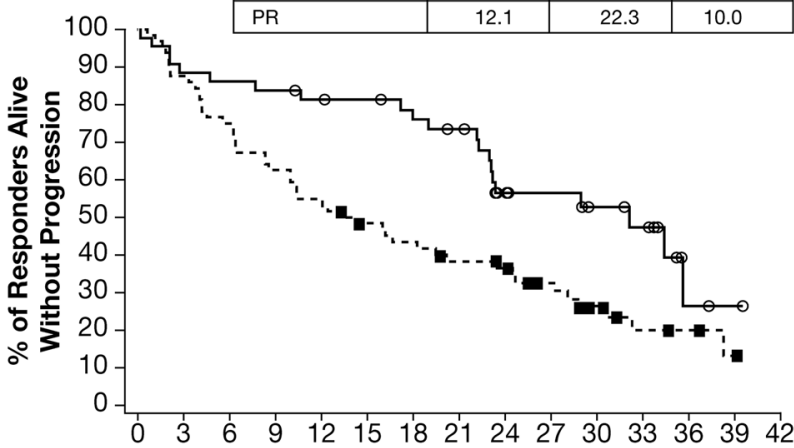

Patients at risk Months

Ibrutinib 1 prior

$\begin{array}{lllllllllllllll}43 & 38 & 37 & 36 & 33 & 32 & 29 & 27 & 17 & 15 & 11 & 9 & 2 & 1 & 0\end{array}$

lbrutinib $>1$ prior

$\begin{array}{lllllllllllllll}64 & 56 & 48 & 40 & 35 & 29 & 26 & 22 & 20 & 15 & 10 & 6 & 4 & 2 & 0\end{array}$
B

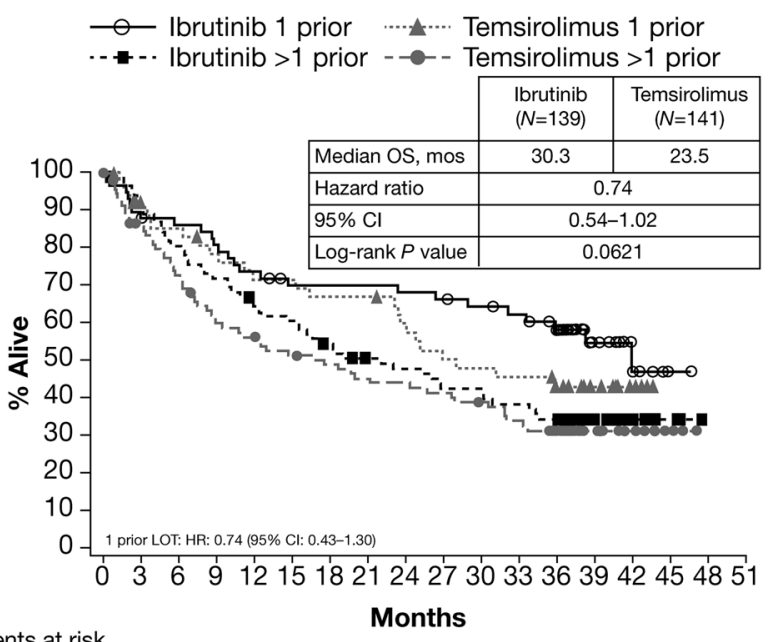

Patients at risk

Ibrutinib 1 prior

$\begin{array}{llllllllllllllllll}57 & 51 & 48 & 45 & 41 & 37 & 37 & 37 & 36 & 35 & 33 & 31 & 27 & 14 & 7 & 1 & 0 & 0\end{array}$ Temsirolimus 1 prior

$\begin{array}{llllllllllllllllll}50 & 42 & 38 & 34 & 31 & 31 & 29 & 29 & 25 & 22 & 20 & 19 & 16 & 11 & 4 & 0 & 0 & 0\end{array}$ lbrutinib $>1$ prior

$\begin{array}{llllllllllllllllll}82 & 74 & 65 & 58 & 51 & 48 & 42 & 37 & 35 & 31 & 31 & 28 & 25 & 14 & 8 & 3 & 0 & 0\end{array}$

Temsirolimus $>1$ prior

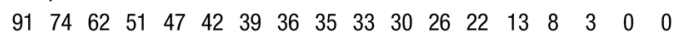

D

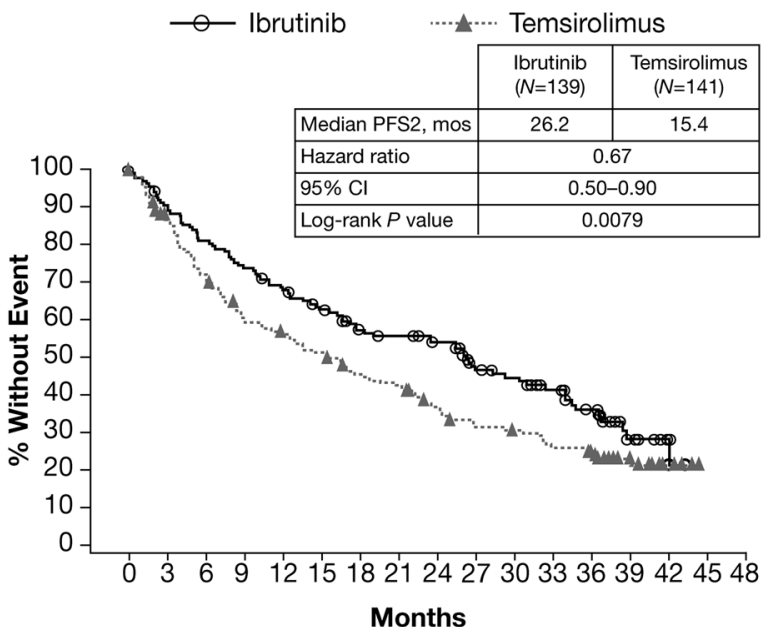

Patients at risk

Ibrutinib $\quad 13912411110192 \quad 82 \quad 71 \quad 68 \quad 63 \quad 47 \quad 43 \quad 34 \quad 25 \quad 11 \quad 4 \quad 0 \quad 0$

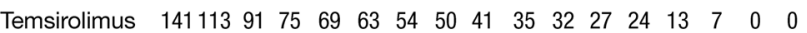

$\mathrm{Cl}$, confidence interval; CR, complete response; DOR, duration of response; ITT, intent-to-treat; OS, overall survival; PFS, progression-free survival; PR, partial response.

Dosing schedule: daily $560 \mathrm{mg}$ of oral ibrutinib (starting on cycle 1, day 1 ) or $175 \mathrm{mg}$ of intravenous temsirolimus (starting on cycle 1 , days 1,8 , 15; then $75 \mathrm{mg}$ on days $1,8,15$ of all subsequent cycles) until disease progression or unacceptable toxicity.

Fig. 1 Efficacy end points in 3-year follow-up in RAY study: a Progression-free survival for ibrutinib and temsirolimus by prior line of therapy; b Overall survival for ibrutinib and temsirolimus by prior

patients who had confirmed disease progression. Fifty-five patients in the temsirolimus group (39\%) received subsequent ibrutinib (42 were included in the formal study crossover; 13 received ibrutinib outside of the study). Disease progression or relapse was the most common reason line of therapy; $\mathbf{c}$ Duration of clinical response by prior line of therapy in patients randomized to ibrutinib; $\mathbf{d}$ Time to second progression or death for ibrutinib and temsirolimus

for discontinuing treatment for both groups (ibrutinib, 78 patients [56\%]; temsirolimus, 66 patients [47\%]). Fewer patients in the ibrutinib group (12 [9\%]) than in the temsirolimus group (39 [28\%]) discontinued treatment due to adverse events (AEs); eight patients in each arm 
Table 1 Treatment-emergent adverse events (AEs) in $\geq 20 \%$ of patients in either treatment arm

\begin{tabular}{|c|c|c|c|c|}
\hline \multirow{2}{*}{$\begin{array}{l}\text { Safety population } \\
\text { AE }(\%)\end{array}$} & \multicolumn{2}{|c|}{$\begin{array}{l}\text { Ibrutinib } \\
(N=139)\end{array}$} & \multicolumn{2}{|c|}{$\begin{array}{l}\text { Temsirolimus } \\
(N=139)\end{array}$} \\
\hline & $\begin{array}{l}\text { Any } \\
\text { grade }\end{array}$ & Grade $\geq 3$ & Any grade & Grade $\geq 3$ \\
\hline \multicolumn{5}{|l|}{ Hematological } \\
\hline Thrombocytopenia & 18.0 & 9.4 & 56.1 & 43.2 \\
\hline Anemia & 19.4 & 8.6 & 43.9 & 20.1 \\
\hline Neutropenia & 15.8 & 12.9 & 26.6 & 17.3 \\
\hline \multicolumn{5}{|l|}{ Non-hematological } \\
\hline Diarrhea & 33.1 & 3.6 & 30.9 & 4.3 \\
\hline Fatigue & 23.7 & 5.0 & 28.8 & 7.2 \\
\hline Cough & 23.0 & 0.7 & 22.3 & 0.0 \\
\hline $\begin{array}{l}\text { Upper respiratory } \\
\text { tract infection }\end{array}$ & 20.1 & 2.2 & 11.5 & 0.7 \\
\hline Pyrexia & 18.7 & 0.7 & 20.9 & 2.2 \\
\hline Nausea & 14.4 & 0.0 & 21.6 & 0.0 \\
\hline Peripheral edema & 13.7 & 0.0 & 23.7 & 2.2 \\
\hline Epistaxis & 9.4 & 0.7 & 23.7 & 1.4 \\
\hline Stomatitis & 2.9 & 0.0 & 20.9 & 3.6 \\
\hline
\end{tabular}

discontinued due to death. Other reasons for discontinuation included refusing further treatment. Median duration of exposure was longer for ibrutinib than temsirolimus (ibrutinib, 14.4 months; temsirolimus, 3.0 months), as in the primary analysis.

Efficacy assessments at primary analysis by the Independent Review Committee showed high concordance with investigator assessment; at final analysis, all efficacy analyses were based on investigator assessment. With additional follow-up, median PFS remained significantly longer for ibrutinib than temsirolimus (15.6 vs. 6.2 months; HR 0.45 [95\% CI 0.35-0.60]; $P<0.0001)$; consistent with the results of the primary analysis [5]. An exploratory post hoc analysis evaluated PFS by number of prior lines of therapy received (ibrutinib, 57 [41\%] 1 prior line and 82 [59\%] >1 prior line; temsirolimus, 50 [35\%] 1 prior line and 91 [65\%] $>1$ prior line). Median PFS for ibrutinib was significantly longer than temsirolimus regardless of the number of prior lines of treatment, and the difference in median PFS between ibrutinib-treated and temsirolimus-treated patients was greatest in those who received 1 prior line of therapy versus $>1$ ( 1 prior line, 25.4 vs. 6.2 months, respectively, HR 0.40 [95\% CI $0.25-0.64]>1$ prior line, 12.1 vs. 6.0 months respectively, HR 0.53 [95\% CI 0.38-0.73]; Fig. 1a).

At the time of final analysis, 77 patients $(55 \%)$ in the ibrutinib group and $83(59 \%)$ in the temsirolimus group had died, with a trend toward improved OS in the patients randomized to receive ibrutinib versus temsirolimus $(30.3$ vs. 23.5 months, respectively; HR 0.74 [95\% CI 0.54-1.02]; $P=0.0621)$. Median OS was longer for ibrutinib than temsirolimus regardless of the extent of prior treatment. However, similar to PFS, a more pronounced OS difference was observed between ibrutinib and temsirolimus treatment in those patients who had received 1 prior line of therapy (1 prior line, 42.1 vs. 27.0 months, respectively, HR 0.74 [95\% CI $0.43-1.30$ ]; $>1$ prior line, 22.1 vs. 17.0 months respectively, HR 0.86 [95\% CI 0.59-1.25]; Fig. 1b).

Overall response rate (ORR) in the final analysis was consistent with the primary analysis $(77 \%$ for ibrutinib vs. 47\% for temsirolimus; odds ratio 4.27 [95\% CI 2.47-7.39]; $P<0.0001$ ), with a higher proportion of patients achieving a complete response (CR) with ibrutinib (23\%) than with temsirolimus (3\%). ORR results for ibrutinib were similar regardless of extent of prior treatment ( $75 \mathrm{vs} .78 \%$ for 1 prior line and $>1$ prior line, respectively). However, the CR rate was two-fold higher in patients treated with ibrutinib who received 1 prior line of therapy than those who received $>1$ prior line: 33 and $16 \%$, respectively. Overall median duration of response (DOR) was 23.1 months $(95 \%$ CI 16.2-28.1) with ibrutinib and 6.3 months (95\% CI 4.7-8.6) with temsirolimus. Patients who achieved a CR on ibrutinib had a longer median DOR than patients who achieved a partial response (PR) $(35.6[N=32]$ vs. 12.1 months $[n=$ 75]; Fig. 1c). While DOR for patients achieving CR with ibrutinib remained consistent regardless of the extent of prior treatment (35.6 $[N=19]$ vs 32.2 months $[N=13]$ for 1 and $>1$ prior line of therapy, respectively), the DOR for patients achieving PR decreased with increasing lines of prior therapy $(22.3[N=24]$ vs. 10.0 months $[N=51]$, respectively, for those who had received 1 vs. $>1$ prior line of therapy). Therefore, DOR for complete responders with only 1 prior line was more than three times longer than for partial responders with $>1$ prior line of therapy.

Consistent with the primary analysis, the most common treatment-emergent AEs (TEAEs) of any grade were diarrhea (33\%), fatigue (24\%), and cough (23\%) in the ibrutinib group, and thrombocytopenia (56\%), anemia (44\%), and diarrhea (31\%) in the temsirolimus group. Despite longer treatment exposure in the ibrutinib group versus the temsirolimus group, the frequency of grade $\geq 3$ TEAEs ( 75 vs. $87 \%$ ), serious AEs of any grade (57 vs. 60\%) and AEs leading to discontinuation (17 vs. $32 \%$ ) were lower in the ibrutinib group than in the temsirolimus group, respectively. The most common grade $\geq 3$ TEAEs for both groups were hematological in nature and were less frequently reported in the ibrutinib group than the temsirolimus group, respectively: neutropenia (13 vs. $17 \%$ ), thrombocytopenia (9 vs. $43 \%$ ) and anemia (9 vs. 20\%) (Table 1). The rate of any grade bleeding was 40 and $33 \%$ in the ibrutinib and temsirolimus groups, respectively. The rate of grade $\geq 3$ bleeding was $9 \%$ in the ibrutinib group and $5 \%$ in the temsirolimus group, with exposure-adjusted rates being lower in the ibrutinib group ( 0.455 events per 100 patient-months $)$ versus 
the temsirolimus group (0.785 events per 100 patientmonths). A higher rate of grade $\geq 3$ atrial fibrillation was observed in the ibrutinib group (5\%) versus the temsirolimus group (1\%); exposure-adjusted rates were similar for both groups ( 0.272 events per 100 patient-months for ibrutinib; 0.221 events per 100 patient-months for temsirolimus).

With longer-term follow-up, the data support a sustained clinical benefit of ibrutinib. Median time to next treatment (TTNT) was longer for patients in the ibrutinib group versus the temsirolimus group ( 31.8 vs. 11.6 months; HR 0.33 [95\% CI 0.24-0.46]; $P<0.0001)$. Moreover, median time from randomization to progression or death after subsequent therapy (PFS2) was longer for ibrutinib than temsirolimus (26.2 vs. 15.4 months; HR 0.67 [95\% CI $0.50-0.90]$; $P=0.0079 ;$ Fig. $1 \mathrm{~d})$.

Nearly half $(N=29 ; 46 \%)$ of 63 patients randomized to ibrutinib who received subsequent anticancer therapy on study were treated with rituximab-based chemotherapy. In these 29 patients, following treatment with ibrutinib, the ORR with rituximab-based chemotherapy was $41 \%$ (24\% CR [ $N=7]$; $17 \%$ PR $[N=5]$ ); response was missing or not evaluable in 11 patients. Fifteen of these 29 patients were treated specifically with bendamustine-rituximab following ibrutinib (ORR 53\%; 40\% CR [ $N=6], 13 \%$ PR $[N=2]$ ); response was missing or not evaluable in six patients.

In conclusion, longer-term follow-up from the final analysis of the RAY study supports the initial report, demonstrating significant improvement in ORR and PFS with ibrutinib over temsirolimus in patients with R/R MCL. At the final analysis, OS showed a trend in favor of ibrutinib versus temsirolimus (30.3 vs. 23.5 months; HR 0.74 [95\% CI $0.54-1.02], P=0.0621)$. In the initial analysis, number of previous lines of therapy was identified as a prognostic factor [5]. With longer follow-up this was evident, with patients who had received 1 prior line of therapy benefiting the most from the use of ibrutinib. More patients were able to achieve a CR (33 vs. $16 \%$ ), and those achieving a PR had a longer DOR (22.3 vs. 10.0 months) when using ibrutinib after 1 versus $>1$ prior line of therapy. In ibrutinib patients with 1 prior line of therapy, this resulted in a doubling of PFS versus ibrutinib patients with $>1$ prior line of therapy (25.4 vs. 12.1 months) and an almost 15-month improvement of OS versus temsirolimus patients with 1 prior line of therapy (42.1 vs. 27.0 months). These data from the RAY study, irrespective of the number of prior lines of therapy, compare favorably to the results from pivotal clinical trials of other single agents in R/R MCL (e.g., bortezomib, lenalidomide, and temsirolimus), the use of which was associated with median PFS of 4-5 months, median OS of 13-19 months, and ORRs of 22-33\% [6-9]. Given that these findings support earlier use of ibrutinib in the relapsed/refractory setting, a relevant clinical question is whether patients can be successfully treated after progression on ibrutinib. Here, we show that patients could be successfully rescued post ibrutinib therapy with rituximab-based chemotherapy (ORR = $41 \%)$, including bendamustine-rituximab $(\mathrm{ORR}=53 \%)$. Importantly, longer follow-up revealed no new late or cumulative toxicities, supporting the overall well-tolerated safety profile for ibrutinib [5]. The significant improvements in PFS2 provide further evidence that ibrutinib benefit is maintained beyond subsequent lines of treatment. Collectively, these results support the role of ibrutinib in the treatment of previously treated MCL. Emerging data suggest that ibrutinib may also have a role in treatment-naïve MCL [10], with multiple phase 3 studies underway (e.g., ENRICH [EudraCT 2015-000832-13], SHINE [NCT01776840], and TRIANGLE [NCT02858258]).

Acknowledgements This study was funded by Janssen Research \& Development. Writing assistance was provided by Juan SanchezCortes, PhD (PAREXEL, Hackensack, NJ, USA) and Natalie Dennis (PAREXEL, Worthing, UK) and was funded by Janssen Global Services, LLC. The authors would like to thank the contribution of Mark Wildgust and Lori Parisi for their support in the long-term reporting for this study. We also thank the patients who participated in this trial, their families and the investigators and coordinators at each of the clinical sites.

Author contributions All authors conceived and/or designed the work that lead to this submission, acquired data and/or played an important role in interpreting the results. All authors were involved in drafting or reviewing the manuscript, and all authors approved the final version of the manuscript.

\section{Compliance with ethical standards}

Conflict of interest Conflicts of interestSR has served as an advisor for Janssen, Pharmacyclics and Napp, and has received research funding from Janssen. WJ has received research funding from Janssen and Pharmacyclics. MJ has received research funding from Janssen, Celgene, Abbvie and Gilead. CR has served as an advisor for Italfarmaco, Teva, Janssen, Takeda and Roche. MT has served as an advisor and received research funding from Janssen. CJ has served as an advisor for Celgene, Janssen, Takeda, Amgen and Roche. MW-H has served as an advisor and received honoraria from Janssen. GH has served as an advisor and received honoraria from Roche, Pfizer, Janssen, CTI and Celgene, and received research support from Roche, Pfizer, Mundipharma, Celgene and CTI. CT has served as an advisor for Bayer, Celgene, Janssen and Roche, and received research funding from Roche. MD has served as an advisor and received research funding from Janssen and Pfizer, and has received honoraria from Janssen. WZ is a contractor of Janssen. TH, JG and JV are employees of Janssen and own stocks in Johnson \& Johnson. The remaining authors declare that they have no conflict of interest.

Open Access This article is licensed under a Creative Commons Attribution 4.0 International License, which permits use, sharing, adaptation, distribution and reproduction in any medium or format, as long as you give appropriate credit to the original author(s) and the source, provide a link to the Creative Commons license, and indicate if changes were made. The images or other third party material in this article are included in the article's Creative Commons license, unless indicated otherwise in a credit line to the material. If material is not 
included in the article's Creative Commons license and your intended use is not permitted by statutory regulation or exceeds the permitted use, you will need to obtain permission directly from the copyright holder. To view a copy of this license, visit http://creativecommons. org/licenses/by/4.0/.

\section{References}

1. Herrmann A, Hoster E, Zwingers T, Brittinger G, Engelhard M, Meusers $\mathrm{P}$, et al. Improvement of overall survival in advanced stage mantle cell lymphoma. J Clin Oncol. 2009;27:512-8.

2. IMBRUVICA ${ }^{\circ}$ [US prescribing information]. Sunnyvale, CA: Pharmacyclics, LLC; Horsham, PA: Janssen Biotech, Inc.; 2017.

3. Wang M, Rule S, Martin P, Goy A, Auer R, Kahl BS, et al. Targeting BTK with ibrutinib in relapsed or refractory mantle-cell lymphoma. N Engl J Med. 2013;369:507-16.

4. Dreyling M, Campo E, Hermine O, Jerkeman M, Le Gouill S, Rule $\mathrm{S}$, et al. Newly diagnosed and relapsed mantle cell lymphoma: ESMO Clinical Practice Guidelines for diagnosis, treatment and follow-up. Ann Oncol. 2017;28:iv62-iv71.

5. Dreyling M, Jurczak W, Jerkeman M, Santucci Silva R, Rusconi $\mathrm{C}$, Trneny $\mathrm{M}$, et al. Ibrutinib versus temsirolimus in patients with relapsed or refractory mantle-cell lymphoma: an international randomised, open-label, phase 3 study. Lancet. 2016;387:770-8.

6. Fisher RI, Bernstein SH, Kahl BS, Djulbegovic B, Robertson MJ, de Vos S, et al. Multicenter phase II study of bortezomib in patients with relapsed or refractory mantle cell lymphoma. J Clin Oncol. 2006;24:4867-74.

7. Goy A, Sinha R, Williams ME, Kalayoglu Besisik S, Drach J, Ramchandren R, et al. Single-agent lenalidomide in patients with mantle-cell lymphoma who relapsed or progressed after or were refractory to bortezomib: phase II MCL-001 (EMERGE) study. J Clin Oncol. 2013;31:3688-95.

8. Hess G, Herbrecht R, Romaguera J, Verhoef G, Crump M, Gisselbrecht $\mathrm{C}$, et al. Phase III study to evaluate temsirolimus compared with investigator's choice therapy for the treatment of relapsed or refractory mantle cell lymphoma. J Clin Oncol. 2009;27:3822-9.

9. Jurczak W, Ramanathan S, Giri P, Romano A, Mocikova H, Clancy $\mathrm{J}$ et al. Comparison of two doses of intravenous temsirolimus in patients with relapsed/refractory mantle cell lymphoma. Leuk Lymphoma. 2018;59:670-8.

10. Wang M, Lee HJ, Thirumurthi S, Chuang HH, Hagemeister FB, Westin JR, et al. Chemotherapy-free induction with ibrutinibrituximab followed by shortened cycles of chemo-immunotherapy consolidation in young, newly diagnosed mantle cell lymphoma patients: a phase II clinical trial. Blood. 2016;128:147.

\title{
Once-weekly carfilzomib, pomalidomide, and low-dose dexamethasone for relapsed/refractory myeloma: a phase I/II study
}

\author{
Sara Bringhen ${ }^{1} \cdot$ Roberto Mina $^{1} \cdot$ Anna Maria Cafro ${ }^{2} \cdot$ Anna Marina Liberati $^{3} \cdot$ Stefano Spada ${ }^{1} \cdot$ Angelo Belotti $^{4} \cdot$ \\ Gianluca Gaidano ${ }^{5}$. Francesca Patriarca ${ }^{6} \cdot$ Rossella Troia $^{1} \cdot$ Renato Fanin ${ }^{6} \cdot$ Lorenzo De Paoli $^{5} \cdot$ Giuseppe Rossi $^{4}$. \\ Alessandra Lombardo ${ }^{7} \cdot$ Paola Bertazzoni ${ }^{2} \cdot$ Antonio Palumbo $^{1} \cdot$ Pieter Sonneveld $^{8} \cdot$ Mario Boccadoro $^{1}$
}

Received: 7 November 2017 / Revised: 21 November 2017 / Accepted: 22 December 2017 / Published online: 30 January 2018

(c) Macmillan Publishers Limited, part of Springer Nature 2018

Antonio Palumbo: Currently Takeda employee.

Electronic supplementary materialThe online version of this article (https://doi.org/10.1038/s41375-018-0024-1) contains supplementary material, which is available to authorized users.

Sara Bringhen

sarabringhen@yahoo.com

1 Myeloma Unit, Division of Hematology, University of Torino, Azienda Ospedaliero-Universitaria Città della Salute e della Scienza di Torino, Torino, Italy

2 Ospedale Niguarda Ca' Granda, Milan, Italy

3 Università degli Studi di Perugia, Struttura Complessa Universitaria Oncoematologia - Azienda Ospedaliera Santa Maria di Terni, Terni, Italy
Despite the progress made in the past two decades, multiple myeloma (MM) remains an incurable disease and the prognosis of patients relapsing after first-generation novel agents is extremely poor [1]. Pomalidomide

4 SC Ematologia e Dipartimento di Oncologia Clinica, A.O. Spedali Civili, Brescia, Italy

5 Division of Hematology, Department of Translational Medicine, University of Eastern Piedmont, Novara, Italy

6 Clinica Ematologica, Azienda sanitaria Universitaria Integrata, DAMe, Università di Udine, Udine, Italy

7 Struttura Complessa Universitaria Oncoematologia - Azienda Ospedaliera Santa Maria di Terni, Terni, Italy

8 Present address: Department of Hematology, Erasmus Medical Center, Rotterdam, Netherlands 